\title{
Effect of Fiber Dispersion and Self-phase Modulation in Multi-channel Subcarrier Multiplexed Optical Signal Transmission
}

\author{
Kyoungsoo Kim, Jichai Jeong, and Jaehoon Lee* \\ Department of Radio Engineering, Korea University, \\ 1, 5Ka, Anam-dong, Seongbuk-gu, Seoul 136-701, Korea
}

(Received July 5, 2010 : revised October 28, 2010 : accepted November 5, 2010)

\begin{abstract}
We investigated the combined effect of fiber chromatic dispersion and self-phase modulation (SPM) in multi-channel subcarrier multiplexed (SCM) optical transmission systems. We theoretically analyzed the transmission characteristics of the SCM signals with the effect of SPM and chromatic dispersion in a single-mode optical fiber by numerical simulations based on the nonlinear Schrödinger equation. The numerical simulation results revealed that the effect of fiber dispersion and SPM could occur independently between subcarrier channels in two-channel SCM systems for small optical modulation index (OMI) and large channel spacing. However, for large OMI, small channel spacing, and large fiber launching power, we found a performance degradation of the two-channel system compared to that of a single-channel system. These parameters are therefore important for the optimization of multi-channel SCM systems applicable to radio over fiber networks.
\end{abstract}

Keywords : Subcarrier multiplexing, Dispersion, Self-phase modulation, Radio-over-fiber

OCIS codes : (060.2330) Fiber optics communications; (060.2360) Fiber optics links and subsystems; (060.4510) Optical communications

\section{INTRODUCTION}

Radio over fiber (RoF) technology has received much interest in order to accommodate demands for increased bandwidth and diversity of services [1-2]. The advantage of RoF technology is the use of an optical fiber with low loss and wide bandwidth for signal transmission. The use of RoF technologies can reduce the size of radio base stations (RBSs) because each RBS needs only a device for optical-to-electrical conversion. Moreover, since the radio modulation unit is located at the control station (CS), the RBSs can use the same frequency band to transmit their radio signals.

RoF technology is often considered one of the simplest solutions to providing future wireless broadband access, and an RoF system including wireless communication systems has recently been proposed [3]. In multi-service environments, the transmission capacity and the number of users in a RoF network can be increased by using sub-carrier multiplexing (SCM) technology that enables multiple RF signals to be transmitted simultaneously over fiber-optic links.
However, when either the subcarrier frequency or the required transmission distance is large, the quality of the signal becomes degraded due to the impairment from the optical fiber channel such as the fiber nonlinearities [4] and the dispersion-induced power penalty (DIPP) that alters the first null point of the fiber transmission response dependent upon the subcarrier frequencies and transmission distance [5]. Although the self-phase modulation (SPM)-induced fiber dispersion compensation effect in millimeter-wave optical transmission systems has been investigated [6,7], the analysis has been limited to transmission systems with a single subcarrier. In this paper, we investigate, for the first time to our knowledge, the combined effect of fiber dispersion and SPM in multi-channel optical SCM transmission systems by theoretical analyses and numerical simulations.

We performed simulations of a two-channel SCM signal and calculated the RF power of the subcarrier signals under various SCM transmission environments, including the effects of fiber dispersion and SPM. From the simulated data, we investigate the effect of the optical modulation index (OMI), fiber launching power, and SCM channel spacing on the

\footnotetext{
*Corresponding author: ejhoon@korea.ac.kr

Color versions of one or more of the figures in this paper are available online.
} 
transmission of SCM signals.

The objective of this paper is to theoretically investigate the combined effect of fiber chromatic dispersion and SPM in optical multi-channel SCM transmission systems. The transmission distance at $P_{3 \mathrm{~dB}}$ after the SMF link with the fiber dispersion and SPM effects can be increased by applying higher fiber launching power into the fiber channel link. However, this gain can be degraded in multichannel environments by the interaction among the subcarrier signals especially at higher fiber launching power. Because it is very difficult to distinguish the origin of these effects in multi-channel environments, we performed numerical simulations using the two-channel SCM signals first to accurately investigate and understand the origin of their interactions.

\section{THEORETICAL ANALYSIS}

Several studies on the feasibility of fiber-induced SPM to compensate for fiber dispersion effects have been reported [6,7]. For a single SCM channel, the nonlinear phase shift induced by SPM at a propagation distance $z$ and normalized time in a frame moving at the group velocity, $T=t-z / v_{g}$ is expressed as [6]:

$$
\begin{aligned}
\Phi_{\text {NL, single }}(z, T) & =-\frac{1-\exp (-\alpha z)}{\alpha} \frac{2 \pi}{\lambda} \frac{n_{2}}{A_{\text {eff }}}\left|A_{\text {single }}(0, T)\right|^{2} \\
& =-\frac{1-\exp (-\alpha z)}{\alpha} \frac{2 \pi}{\lambda} \frac{n_{2}}{A_{\text {eff }}} P_{0}\left(1+m_{i} \cos \left(2 \pi f_{m} t\right)\right)
\end{aligned}
$$

with the field envelope at the fiber input, $A_{\text {singe }}(0, T)=$ $\sqrt{P_{0}} \sqrt{1+m_{i} \cos \left(2 \pi f_{m} t\right)}$ where $P_{0}$ is the optical power, $m_{i}$ is the optical modulation index, $f_{m}$ is the RF subcarrier frequency, $\alpha$ is the fiber attenuation coefficient, $n_{2}$ is the nonlinear index coefficient, $A_{\text {eff }}$ is the effective core area of the fiber, and $\lambda$ is the wavelength of the optical carrier.

For an SCM signal with two RF subcarrier channels (shown in Fig. 1), the initial pulse envelope and the nonlinear phase shift induced by SPM can be expressed as:

$$
\begin{aligned}
A_{2 \mathrm{SCM}}(0, T) & =\sqrt{P_{0}} \sqrt{1+m_{i} \cos \left(2 \pi f_{m} t\right)+m_{i} \cos \left(2 \pi f_{n} t\right)} \\
\Phi_{\text {NL.2SOM }}(z, T) & =-\frac{1-\exp (-\alpha z)}{\alpha} \frac{2 \pi}{\lambda} \frac{2 \pi}{A_{\text {cff }}}\left|A_{\text {scen }}(0, T)\right|^{2} \\
& =-\frac{1-\exp (-\alpha z)}{\alpha} \frac{2 \pi}{\lambda} \frac{n_{2}}{A_{\text {cff }}} P_{o}\left(1+m_{i} \cos \left(2 \pi f_{n} t\right)+m_{i} \cos \left(2 \pi f_{n} t\right)\right)
\end{aligned}
$$

where $f_{m}$ and $f_{n}$ are the two different RF subcarrier frequencies. We consider only two SCM channels in order to simplify the problem and minimize the computational expense.
The field of the signal with a single RF subcarrier, considering solely the effect of SPM, can be expressed as:

$$
\begin{aligned}
A_{\text {single }}(z, T) & =A_{\text {single }}(0, T) \cdot \exp (-\alpha z / 2) \cdot \exp \left(-j \Phi_{N L, 2 s c M}(z, T)\right) \\
& =\sqrt{P_{0}} \sqrt{1+m_{i} \cos \left(2 \pi f_{m} t\right)} \cdot \exp (-\alpha z / 2) \\
& \cdot \exp \left[j \cdot \frac{1-\exp (-\alpha z)}{\alpha} \frac{2 \pi}{\lambda} \frac{n_{2}}{A_{\text {eff }}}\left(P_{0}+P_{0} \cdot m_{i} \cos \left(2 \pi f_{m} t\right)\right)\right]
\end{aligned}
$$

and the field of SCM signal with two RF subcarrier channels, considering the initial pulse envelope and the phase shift due to SPM, is expressed as:

$$
\begin{aligned}
& A_{\text {scom }}(z, T)=A_{\text {scsen }}(0, T) \cdot \exp (-\alpha z / 2) \cdot \exp \left(-j \Phi_{\text {N.2.2Scur }}(z, T)\right) \\
& =\sqrt{P_{0}} \sqrt{1+m_{i} \cos \left(2 \pi f_{n} t\right)+m_{i} \cos \left(2 \pi f_{n} t\right)} \cdot \exp (-\alpha z / 2) \\
& \cdot \exp \left[j \cdot \frac{1-\exp (-\alpha z)}{\alpha} \frac{2 \pi}{\lambda} \frac{n_{2}}{A_{\mathrm{ctf}}}\left(P_{0}+P_{0} \cdot m_{i} \cos \left(2 \pi f_{m} t\right)+P_{0} \cdot m_{i} \cos \left(2 \pi f_{n} t\right)\right)\right]
\end{aligned}
$$

The last term in Eq. (4) describes the phase shift induced by the SPM effect. It is well known that, in single channel SCM transmission system, the SPM-induced frequency chirp can compensate for the pulse distortion due to chromatic dispersion in a single-mode fiber (SMF) $[6,7]$. However, in two-channel SCM transmission system, there is additional phase shift caused due to the other SCM frequency $f_{n}$, as shown in Eq. (5). The additional phase shift depends on the optical modulation index, the fiber launching power, and the frequency. Therefore, there is some interaction between the different RF subcarrier channels, $f_{m}$ and $f_{n}$, and this interaction can reduce the fiber dispersion compensation effect due to SPM-induced frequency chirp. The addition of extra SCM channels will cause more phase shift of the entire SCM signal envelope, as can be seen from the exponential term in Eq. (5). Therefore, the interaction among the subcarrier signals will be increased and the degradation of the SPM-induced fiber dispersion compensation effect will also be increased.

\section{SIMULATION METHOD}

As shown in Fig. 1, the RF subcarrier signals were modulated by an optical linear modulator in order to observe the effect of fiber transmission. The lasing wavelength was $1550 \mathrm{~nm}$, and the electrical RF subcarrier signals were sinusoids. For an optical signal propagating in a nonlinear, dispersive, and lossy single-mode fiber, the evolution of a slowly varying electric field pulse envelope can be found from the nonlinear Schrödinger equation [8]. We performed numerical simulations to examine the combined effect of fiber dispersion and SPM by using the split-step Fourier method $[8,9]$. In order to vary the strength of the SPM effect, the fiber launching power entering the SMF 


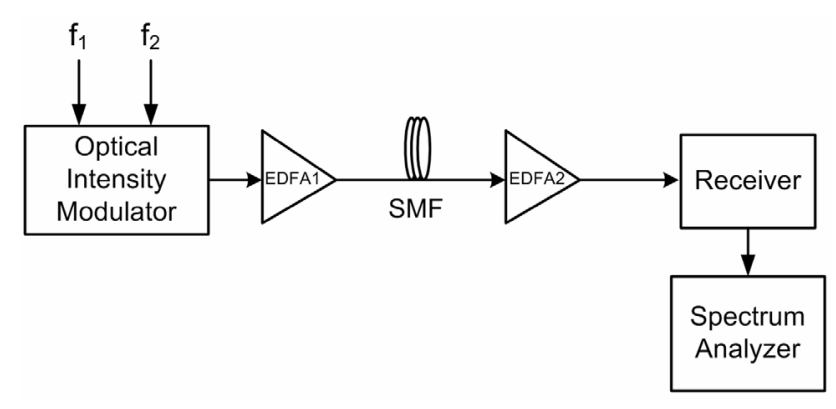

FIG. 1. Schematic diagram of the SCM transmission link.

TABLE 1. Parameters used in the numerical simulation.

\begin{tabular}{l|l}
\hline \hline SCM sub-carrier frequency & $5,5.1,5.5,7,9 \mathrm{GHz}$ \\
\hline SCM channel configuration & Single, 2 channel \\
\hline Lasing wavelength & $1550 \mathrm{~nm}$ \\
\hline Fiber dispersion coefficient $(=D)$ & $17 \mathrm{ps} / \mathrm{nm} / \mathrm{km}$ \\
\hline Fiber attenuation coefficient & $0.22 \mathrm{~dB} / \mathrm{km}$ \\
\hline Fiber effective core area & $78 \mu \mathrm{m}^{2}$ \\
\hline Fiber nonlinear index coefficient & $3.0 \times 10^{-20} \mathrm{~m}^{2} / \mathrm{W}$ \\
\hline Fiber length & $0 \sim 150 \mathrm{~km}$ \\
\hline Fiber launching power & $0 \sim 10 \mathrm{dBm}$ \\
\hline
\end{tabular}

was adjusted between 0 and $10 \mathrm{dBm}$ using an optical amplifier before transmission. The length of the SMF was varied from 0 to $150 \mathrm{~km}$ in order to observe different fiber dispersion effects. We used the electrical power spectra calculated after detection with a photodiode to calculate the RF carrier power of each SCM channel. The specific parameters of the optical fiber link used in our simulations are shown in Table 1 .

\section{NUMERICAL SIMULATION RESULTS}

First, we simulated two-channel SCM transmission systems with a large channel spacing, and investigated the combined effect of fiber dispersion and SPM. Two subcarrier signals at $5 \mathrm{GHz}$ and $9 \mathrm{GHz}$ were combined. The OMI per channel was $20 \%$ and the detected RF carrier power for each channel was calculated after transmission over an SMF link up to $120 \mathrm{~km}$. We also simulated SCM systems with a single subcarrier for comparison. Fig. 2 illustrates the detected RF carrier power for the single- and two-channel signals. Due to the DIPP characteristic, the null point of the carrier power fading as a function of the transmission distance appeared first for the $9 \mathrm{GHz}$ signal [5]. When the higher fiber launching power $(10 \mathrm{dBm})$ was applied, the transmission distance at the same detected RF carrier power was extended for $5 \mathrm{GHz}$ and $9 \mathrm{GHz}$ signals. This is due to the fact that the chirping effect of SPM combines with the fiber dispersion, leading to dispersion compensation in

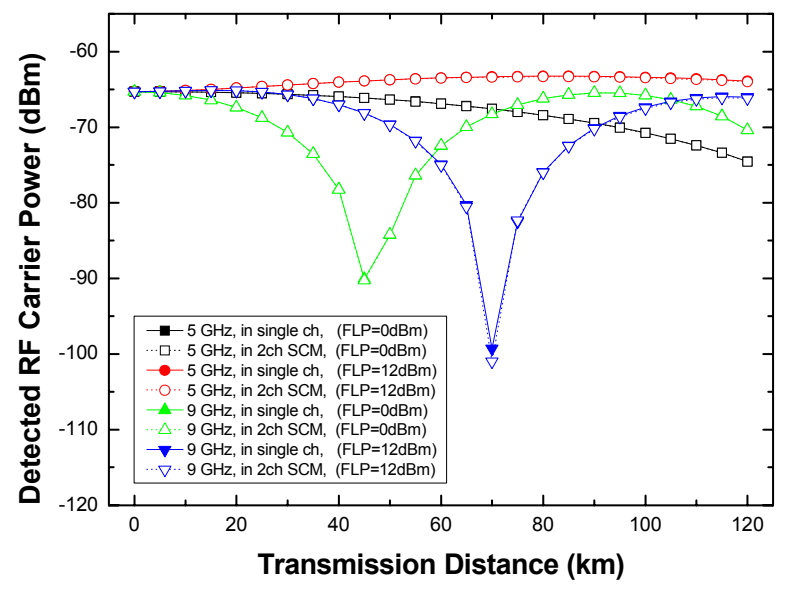

FIG. 2. Detected RF carrier power versus transmission distance for single- and two-channel SCM signals; $5 \mathrm{GHz}$ and $9 \mathrm{GHz}$.

the SMF transmission link [6, 7].

However, the detected RF carrier powers for the singleand two-channel SCM signals were almost the same at the same subcarrier frequency and fiber launching power for all transmission distances. This is because the OMI per channel was relatively small and the channel spacing between the subcarrier channels was large. Therefore, there was little interaction between the subcarrier signals. The intermodulation product near two SCM channels was very small, even for an OMI per channel of $50 \%$; therefore it can be expected that the effect of the nonlinear distortion after photo-detection in the receiver was also small.

We evaluated the transmission distances at which the detected RF carrier power dropped by $3 \mathrm{~dB}$. These values are termed the "transmission distance at $P_{3 \mathrm{~dB}}$ " in this paper. The value of $P_{3 \mathrm{~dB}}$ was almost the same for different subcarrier frequencies; however, the RF carrier power fading curves varied with subcarrier frequency and fiber launching power. Therefore, the calculated transmission distance at $P_{3 \mathrm{~dB}}$ also changed. As can be seen from the additional phase shift term in Eq. (5), the fiber launching power, OMI per channel, and SCM channel spacing are important parameters for evaluating the entire SCM transmission system performance. In order to confirm the effect of these parameters, the frequency of channel 1 was fixed at $5 \mathrm{GHz}$ and the frequency of channel 2 was varied between 5.1 and $9 \mathrm{GHz}$. Two different simulation cases were considered. First, single- and two-channel SCM systems with an OMI per channel of $20 \%$ were evaluated in terms of the transmission distance at $P_{3 \mathrm{~dB}}$. Secondly, a performance evaluation was carried out again with an OMI of $50 \%$ in order to investigate the effect of OMI per SCM channel.

Fig. 3(a) shows the transmission distance at $P_{3 \mathrm{~dB}}$ for the subcarrier signals at $5 \mathrm{GHz}$ with an OMI per channel of $20 \%$. The transmission distance at $P_{3 \mathrm{~dB}}$ increased with larger fiber launching power due to the dispersion compensation effect in combination with SPM. The calculated 


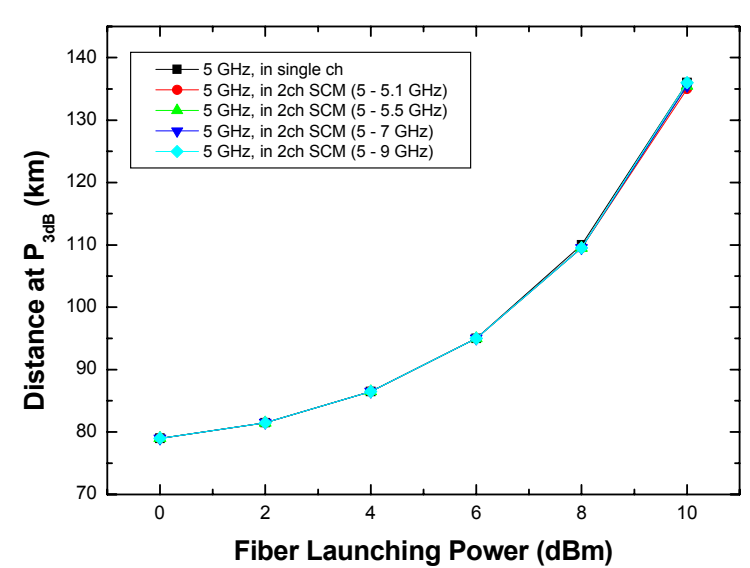

(a)

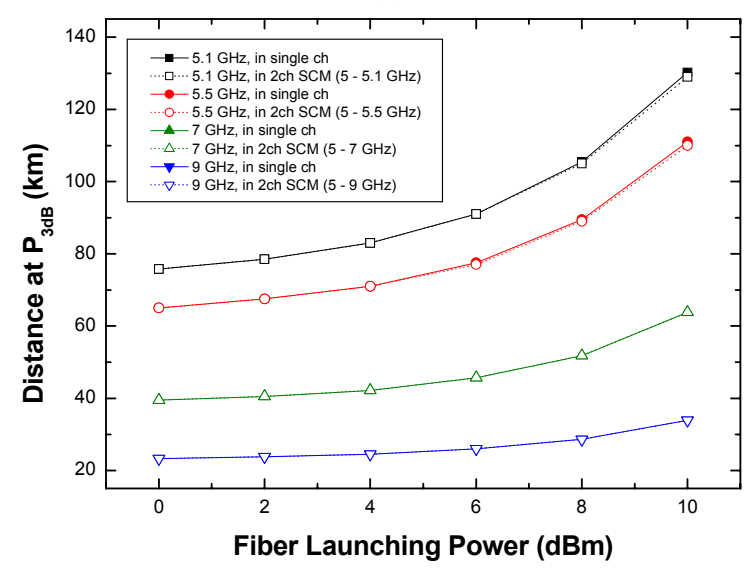

(b)

FIG. 3. Transmission distance at $P_{3 \mathrm{~dB}}$ for the SCM signals with 20\% OMI per channel. (a) Channel 1 (5 GHz) and (b) channel $2(5.1-9 \mathrm{GHz})$.

values were almost the same for the single- and two-channel configurations, even for different SCM channel allocations. Fig. 3(b) shows the transmission distance at $P_{3 \mathrm{~dB}}$ as a function of fiber launching power when channel 2 was varied from 5.1 to $9 \mathrm{GHz}$, and with an OMI per channel of $20 \%$. As the frequency of channel 2 increased, the transmission distance at $P_{3 \mathrm{~dB}}$ fell due to the DIPP characteristic of the SMF link. Based on these simulation results, we can infer that the SCM channel spacing and fiber launching power do not result in serious performance deterioration for small values of the OMI per channel. It was shown that the performance of two-channel SCM systems was almost equal to that of single channel systems. This also follows from Fig. 2, because the combined effect of fiber dispersion and SPM occurred independently between the two SCM channels for small values of OMI per channel.

Fig. 4(a) shows the transmission distance at $P_{3 \mathrm{~dB}}$ of channel 1, and Fig. 4(b) shows the transmission distance at $P_{3 \mathrm{~dB}}$ for channel 2, with an OMI per channel of $50 \%$. The transmission distance at $P_{3 \mathrm{~dB}}$ increased with increasing fiber launching power due to SPM-induced dispersion compen-

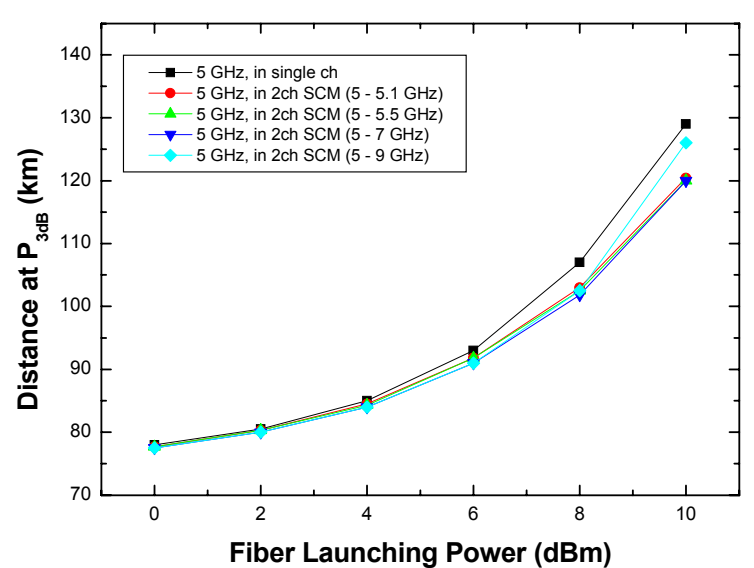

(a)

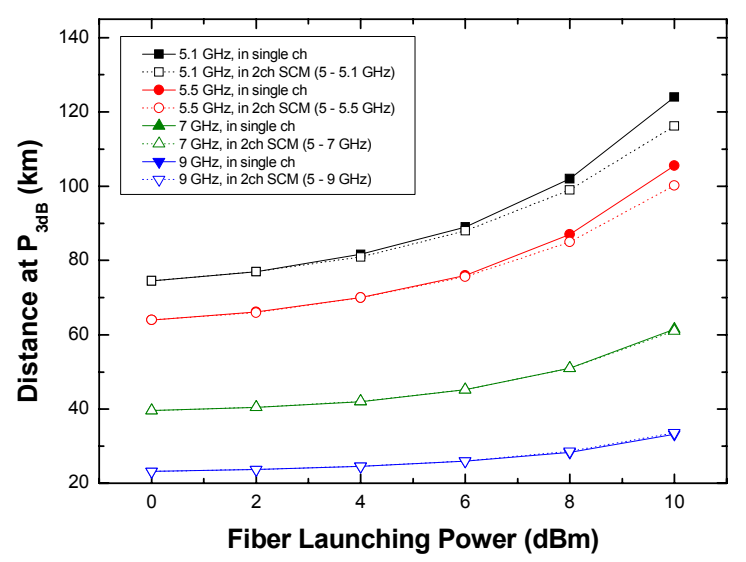

(b)

FIG. 4. Transmission distance at $P_{3 \mathrm{~dB}}$ for the SCM signals with 50\% OMI per channel. (a) Channel $1(5 \mathrm{GHz})$ and (b) channel $2(5.1-9 \mathrm{GHz})$.

sation effect. However, the two-channel system showed a drop in the transmission distance at $P_{3 \mathrm{~dB}}$ compared to the performance of the single channel configuration. Therefore, the addition of another SCM channel reduced the fiber dispersion compensation effect caused by SPM.

From the analysis in Section 2, when the SCM signals have large OMI per channel and the channel spacing is small, the effect of the additional phase shift term in Eq. (5) is more pronounced. Therefore, the performance improvement due to the interaction between fiber dispersion and SPM can be reduced at high fiber launching powers.

Fig. 5(a) and (b) show the contour plots of the transmission distance at $P_{3 \mathrm{~dB}}$ for the first SCM channel at 5 $\mathrm{GHz}$ when the second channel is at (a) $5.1 \mathrm{GHz}$ and (b) $9 \mathrm{GHz}$, respectively. Up to the OMI of $15 \%$, there is no significant difference in the transmission distance at $P_{3 \mathrm{~dB}}$ with varying the fiber launching power from 5 to $10 \mathrm{dBm}$ because the interaction between the subcarrier signals is relatively small. However, when the OMI per channel is increased over $15 \%$, the calculated value of the transmission distance at $P_{3 \mathrm{~dB}}$ of the SCM signal with the SCM channel 


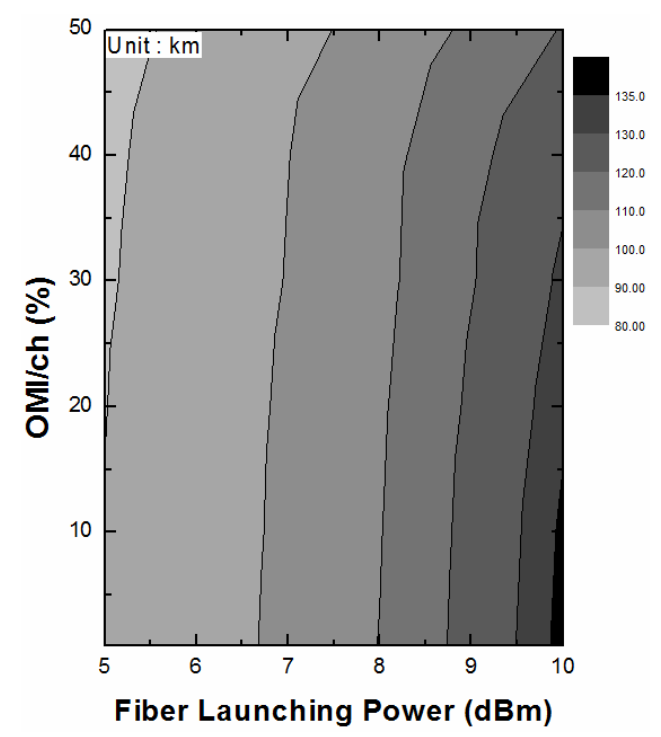

(a)

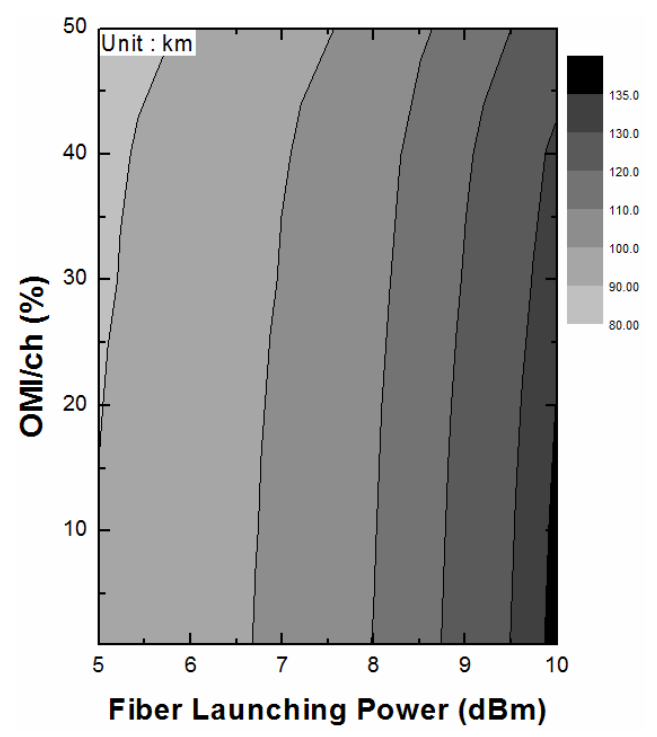

(b)

FIG. 5. Contour plots of Transmission distance at $P_{3 \mathrm{~dB}}$ calculated from the first SCM channel fixed at 5 GHz while the second SCM channel is varied at (a) $5.1 \mathrm{GHz}$ and (b) $9 \mathrm{GHz}$.

spacing of $100 \mathrm{MHz}$, as shown in Fig. 5(a), is degraded especially in the higher fiber launching power because the increased interaction between subcarrier signals can reduce the effect of SPM-induced fiber dispersion compensation. For example, when the OMI of $40 \%$ and the fiber launching power of $10 \mathrm{dBm}$ are applied in the system, the SCM system with $100 \mathrm{MHz}$ channel spacing (Fig. 5(a)) cannot guarantee the transmission distance at $P_{3 \mathrm{~dB}}$ over $130 \mathrm{~km}$, whereas the $\mathrm{SCM}$ system with $4 \mathrm{GHz}$ channel spacing (Fig. 5(b)) can guarantee the performance. Therefore, we can suggest that the OMI should be smaller than $15 \%$ to provide similar transmission performance when the channel spacing is larger than $100 \mathrm{MHz}$ and the fiber launching power is less than $10 \mathrm{dBm}$.

\section{CONCLUSION}

We investigated the combined effect of fiber dispersion and SPM in optical SCM transmission systems. From a theoretical analysis and numerical simulations, we found that the dispersion compensation effect in combination with SPM occurred independently for each subcarrier for a small OMI per channel and large channel spacing.

We evaluated the performance of single-channel and two-channel SCM systems in terms of the transmission distances at $P_{3 \mathrm{~dB}}$. The simulated data showed that the dispersion compensation effect due to SPM increased for high fiber launching powers for both cases. However, the transmission distances at $P_{3 \mathrm{~dB}}$ of the two-channel SCM system was reduced compared to that of the single-channel system, especially at higher fiber launching powers and smaller SCM channel spacing. This resulted from the increased interaction between subcarrier channels due to the effect of an additional phase shift caused by the other channel. This interaction reduced the SPM-induced fiber chromatic dispersion compensation effect in multi-channel SCM systems. This result can be used for the optimization of multi-service systems using SCM technology in future RoF networks.

\section{ACKNOWLEDGMENT}

This work was supported in part by the Second Brain Korea 21 project in 2010 and Basic Science Research Program through the National Research Foundation of Korea(NRF) funded by the Ministry of Education, Science and Technology(2010-0013566).

\section{REFERENCES}

1. K. Morita and H. Ohtsuka, "The new generation of wireless communication based on fiber-radio technologies," IEICE Trans. Commun. E76-B, 1061-1067 (1993).

2. M. K. Hong, S. K. Han, and S. H. Lee, "Linearization of DFB LD by using cross gain modulation of reflective SOA in radio-over-fiber link," J. Opt. Soc. Korea 11, 158-161 (2007).

3. S. T. Hwang, H. Kim, B. J. Kim, S. K. Kim, J. H. Lee, H. L. Lee, Y. G. Kim, G. W. Lee, S. H. Kim, and Y. J. Oh, "RoF technologies for in-building wireless systems," IEICE Trans. Electron. E90-C, 345-350 (2007).

4. H. D. Jang, K. S. Kim, J. H. Lee, and J. C. Jeong, "Transmission performance of $40 \mathrm{~Gb} / \mathrm{s}$ PM duobinary signals due to fiber nonlinearities in DWDM systems using VSB filtering techniques," J. Opt. Soc. Korea 13, 354-360 (2009). 
5. J. M. Fuster, J. Marti, J. L. Corral, V. Polo, and F. Ramos, "Generalized study of dispersion-induced power penalty mitigation techniques in millimeter-wave fiber-optic links," IEEE J. Lightwave Technol. 18, 933-940 (2000).

6. F. Ramos, J. Marti, V. Polo, and J. M. Fuster, "On the use of fiber-induced self-phase modulation to reduce chromatic dispersion effects in microwave/millimeter-wave optical systems," IEEE Photon. Technol. Lett. 10, 1473-1475 (1998).

7. F. Ramos, J. Marti, V. Polo, and J. M. Fuster, "Dispersiontolerant data transmission based on the use of fiber-induced self-phase modulation in microwave optical links," Microwave Opt. Technol. Lett. 27, 1-4 (2000).

8. G. P. Agrawal, Nonlinear Fiber Optics (Academic Press, New York, USA, 1989).

9. J. H. Lee, S. K. Kim, Y. G. Kim, O. Mizuhara, G. Y. Lyu, S. S. Kang, J. H. Song, and J. C. Jeong, "Performance evaluation and prediction using eye margin characteristics for optical transmission systems," Opt. Comm. 193, 113-120 (2001). 